\title{
Building Knowledge Bases for the Generation of Software Documentation *
}

\author{
Cécile Paris ${ }^{\dagger}$ and Keith Vander Linden \\ ITRI, University of Brighton \\ Lewes Road \\ Brighton BN2 4AT, UK \\ $\{c l p, k n v l\} @ i t r i . b r i g h t o n . a c . u k$
}

\begin{abstract}
Automated text generation requires a underlying knowledge base from which to generate, which is often difficult to produce. Software documentation is one domain in which parts of this knowledge base may be derived automatically. In this paper, we describe JRAFTER, an authoring support tool for generating usercentred software documentation, and in particular, we describe how parts of its required knowledge base can be obtained automatically.
\end{abstract}

\section{Introduction}

Automated text generation is becoming an attractive technology because it allows for the generation of text in different styles and in different languages from a single underlying knowledge base. The well-known problem with the technology is that this knowledge base is often difficult to build. In most research generation systems, this knowledgo base is essentially built by hand. No genoral solution to this problem has been proposed because each application has its own domain specific requirements.

It is clear, however, that for text generation technology to become viable, there must be some

\footnotetext{
* This work is partially supported by the Enginocring and Physical Sciences Research Council (EP. Sic) Grant J19221, by BC/DAAD ARC Project 293, by the Commission of the European Union Grant LRE-62009, and by the Office of Naval Research Grant N00014-96-1-0465.

† Starting this Fall, Dr. Paris' address will be CSIRO, Division of Information Technology, Sydney Laboratory, Building E6B, Macquarie University Campus, North Ryde, Sydney, NSW 2113, Australia

$\ddagger$ Starting this Fall, Dr. Vander Linden's address will be Dept. of Mathematics and Computer Science, Calvin College, Grand Rapids, MI 49546, USA.
}

way to obtain at least portions of the knowledge basc automatically. There could be a program which automatically derives the knowledge basc or perhaps the knowledge base could be built as part of manual processes that would have to be performed anyway. Either way, the marginal cost of adding text generation would be greatly reduced.

In this paper, we show that software documentation is an attractive application for multilingual text gencration because it is an arca in which pre-built knowledge bases are becoming available. This is due in large part to the advancements in the user interface design community which we will revicw first. We then discuss the nature of the knowledge base required for the greneration of documentation and how parts of it might be derived automatically. Finally, wo illustrate this idea nusing DRAF'TER, a support tool for generating multilingual software documentation.

\section{Background}

Researchers in user interface design have started to build tools which produce both code and documentation. These tools tend to be based on a central model of the interface under development, the interface model, a formal represontation which can be used not only for code generation but also for document generation, c.g., (Puerta and Szckcly, 1994; Moriyon et al., 1994). Moriyon et al (1994), for example, have used the interface model in the generation of on-line help. Their help messages indicate the actions a user can perform in a particular situation and what would result from those actions. They report, however, that task-oriented help is beyond the capabilitios of their system; task-oriented holp would indicate why the user might want to perform any of the actions that are available.

In general, however, the documentation produced by these systems is limited in two main ways: it docs not correspond to task-oriented documentation, which is, however, what end-users re- 
quire and it is usually based on simple template generation, which does not allow flexibility with regard to the style of the text produced or the language that is used. 'These limitations stem, on the one hand, from the fact that interface modcls in general contain system-oriented information (e.g., what happens when a button is pushed) but not task-oriented information (e.g., why one might, want to push the button), and, on the other hancl, from the focus of the rescarch, that is system and interface design and not natural language generation.

In the DR $\mathrm{Dl}$ "TER project, we have atitempted to address these two issues. Wo address the first by providing tools that allow technical authors to build richer interface models. These richer models integrate task information into the information already available in interface models. 'This task information, which is commonly found in task modcls, e.g., GOMS (Card et al., 1983), supports the production of user-centred docmentation. We address the second by providing more general text gencration facilities which support multiple styles and multiple languages.

\section{Representing the users' tasks}

Farly in the 1)RAFrer project, we conducted intervicws with technical anthors (mostly software documentation specialists) in order to understiand the documentation process as it, currently exists, to see if an authoring tool would be holpful, and if so how it might, be used. We found that technical authors start the documcntation process by learning how to use the interface in question, constructing a user-oriented mental model of the product. They frequently have no input other than the software itself. The authors indicated that they would welcome tools to help them collect the appropriate information and create a formal representation of the resulting model. Such a representation would support iterative construction of the documentiation and information reuse.

Building our drafting tool, therefore, required us first to determine how to represent the model of a task, and then to build tools for creating and manipulating this model. Given that the general structure of instructional texts is hicrarchical, we chose a represcntation that expresses a hicrarchy of goals and sub-goals. The representation is thus sirnilar to the traditional structures found in AI planning, e.g., (Sacerdoti, 1977), and also to task models used in interface design, e.g., (Card ot al., 1983). Becanse user documentation frequently includes information other than the raw actions to be performed, our representation allows authors to include information not typically found in traditional plan representations such as: useroriented motivational goals, helpful side-cflects, and general comments.

As an example, consider the representiation of a sub-set of the procedure for saving a new file in a Microsoft Word-like editor shown in ligure 1. The oval boxes in the figure represent actions and the rectangles represent plans. Each of the action nodes in this structure represent intercomected complexes of procedural and descriptive instances. For example, the main user goal of saving a document, represented in the figure by the action nocle "Save a Document", is implemented in the knowledge base as a complex of instances representing the action being performed (in this case saving), the agent, who performs action (the reader), the pationt on whom the action is performed (the current documenti), ctc. $\Lambda \mathrm{ll}$ of this information is required to gencrate expressions of the action, but presenting it would overly complicate the graph.

The links actually shown in the figure are based on the procedural relations in the domain model. For example, the plan for saving a document (Save--Document-Plan) is linked to its goal (Save $\Lambda$ Document), to its precondition (Open-Save$\Lambda$ s), and to its sub-actions of typing a name for the current document. ('Type-Docmuent-Name), opening the folder in which it is to be saved (Openlolder), and clicking the Save button (ChooseSave-I3utton). The procondition (Open-Save-As) must be performed before the sub-steps may be attompted and is in turn linked to furthor sub-plans (Choosing-Plan and Clicking-Plan). This indicates that the Savo-As dialog box may be opened by either choosing the Save option from the file mem (Choose-Save-Option) or clicking the Save button on the tool bar (Click-Save-Icon).

This task model represents the procedures that a $u$ ser might perform when using an application and is the basis for generating user-centred documentation, such as one of DRAFrere's texts shown in Figure 4. It includes the users' high-level goals (e.g., "save a document") as well as their lowlevel interface manipulations ("choose the save buttion").

\section{Input from the Design Process}

In our earlier work, we provided tools that supported the construction of the task model by hand (Paris et al, 1995). 'This went some way to antdressing the technical authors' desire for a formal model and tools to build it. Building the model from scratch, however, even with the help of our menu based interface, was a tedious and lengthy 


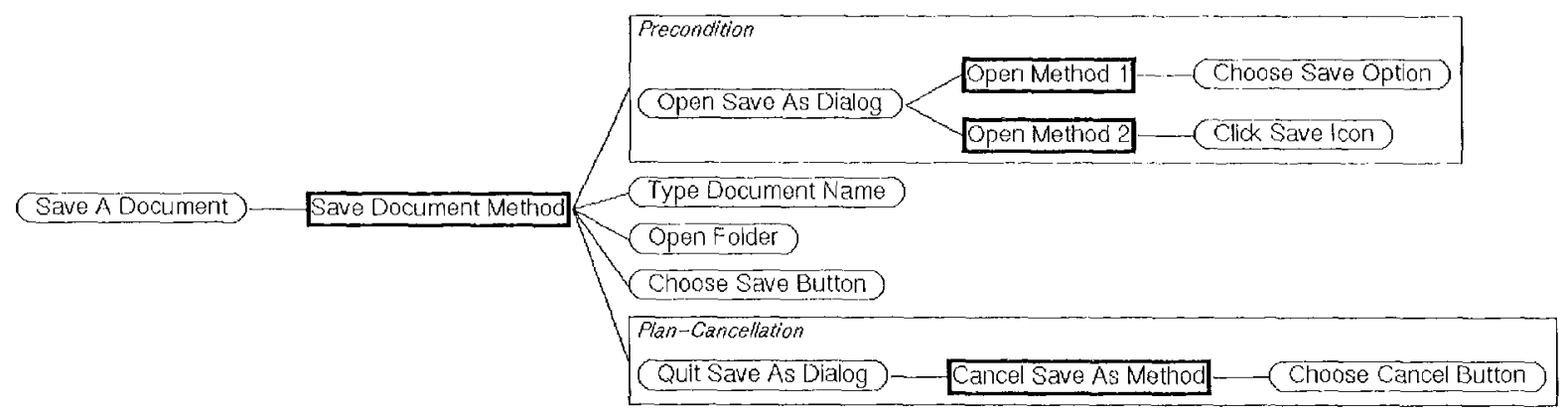

Figure 1: The Saving Procedure Graph

process which could potentially have rendered the DRAFTrR system impractical. There was a clear need for facilities to ease the input task. In line with this, we noticed that certain elements of the model were also present in the specifications developed in user interface design environments. Indeed, we found that a number of the actions and objects in the model could be automatically acquired from a design tool, thus providing basic building blocks from which the full model could be constructed.

To illustrate this idea, we have built our example document editor application in VisualWorks, a widely available interface design environment (Vis, 1994). This tool allows one to define the windows, dialog boxes, and other widgets relevant for the application under development, and produces a prototype of the interface thus specificd. Its output also includes declarative specifications of all the widgets. These specifications are thus available to be exploited by other systems. In particular, we found that these specifications could be readily transformed into a form appropriate for the knowledge base required by a text generation system such as DRAF'IrR. In our example then, wo build a VisualWorks mock-up of our word processing application, and DRAFr'Tr derives task model instances for all the windows and widgets in the application (e.g, the Save-As dialog box and all its widgets) directly from the SmallTalk source code. DR FT'wR is also able to infer the basic interface actions that can be performed on the various interface widgets and creates task model instances for them as well. For example, the system automatically defines a clicking action instance for any "button" on the interface. Similarly, it creates opening and closing actions for all "windows".

Although this set of instances does not represent all the information that could, in principle, be derived from the SmallTalk specifications of the editor application, it nevertheless simplifies greatly the technical author's task of knowledge specification by providing the building blocks from which higher-level procedures can be defined. In the case of our adnititedly simple example, seven of the nine actions in the procedural structure are automatically specified. The author is required to specify only the main user goal action and the three plan nodes. This is, therefore, a step towards automatically building the knowledge base required for the generation system. It is also a step towards integrating the design and documentation processes, which is now widely recognised as being desirable. In our current work, we are investigating how more of the design knowledge can be made accessible and understandable to the toch nical authors, and what other tools would further facilitate the authors' task. We are also looking at a tighter integration of the design and documentation processes, one in which the individuals involved work together during design.

\section{DRAFTER}

We now describe DRAFr'sR, a technical authoring tool which supports the construction of the task model discussed above and the drafting of multilingual instructions from that model. We will focus on how it supports the author in augmenting the information automatically acquired from the interface design tool. IDRAFTER's general architecture, shown in Figure 2, is based on two main processing modules:

- The Author Interface (shown on the far: left of the diagram) allows authors to build a task model and to control the drafting process.

- The Drafting Tool (shown on the far right of the diagram) comprises two major components: the Text Planner and the Tactical Generator. The Text, Planner determines the content and structure 


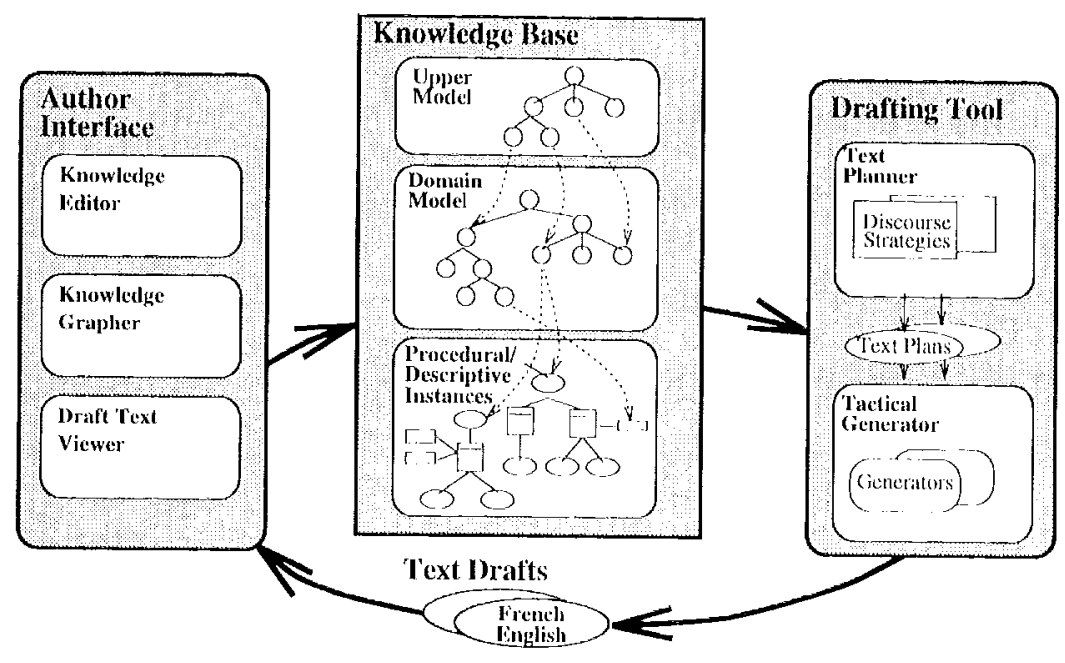

Figure 2: J)ataflow in DRA "rER

of the text as woll as the detailed struc ture of the sentences therein. The 'Lactical Gonorator performs the surface realisation of the sentences.

The knowledge base (in the middle of the figure) underlies the task model built, by the technical anthor. 'The Drafting 'lool takes this representation as input and produces English and French draftis of the appropriate tutorial instructions. In this section we detail each of these components in the context of an eximple.

\subsection{The Knowledge Base}

The knowledge base supportis the construction of the task modol discussed above. It is an hiowarchical structure implemented in 1.00M (MacGregor, 1988). The root is the Penman Morged Upper Model (13ateman, 1995), an ontology of distinctions relevant in expressing actions, objects, and qualities in natural language. The knowledge base contains further layers corresponding to: (1) the concepts and relations general to all instructions; (2) those general only to software interfaces; and (3) those specific to the chosen software applicattion domains (in our case text processing tools).

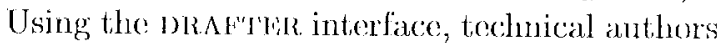
specify hicrarchical task models, such as the one shown in Figure 1, by building nodes and conneeting them with the appropriate procedural relations. The low-levol building blocks of the task model are derived antomatically, and DRAFr's allows the technical author to connect them and add higher-level task information as appropriate, using an interface based on controlled language and the use of menus to guide the author.

\subsection{The Interface}

DRA R'TR's interface is implemented in cu, and includes the following modules:

- 'Iho Knowledge Liditor allows tho author to comstruct and maintain the procedural representation;

- The Knowledge Grapher allows the all thor to visualise the hiorarchical structure of the procelural representiation;

- The Draft Text Viewer allows the author to view and edit the antomatically generated English and Finch clrafts.

These functions an be invoked from menus or from monse-sensitive objects in a style common to systems such as Motif.

\subsubsection{The Knowledge Editor}

This tool makes the structure of the knowledge base on which the task model is built mone accessible to the author. It allows the author to perform two basic tasks: (1) specifying the ac tion nodes appearing in the structure and not yet derived from the interface designed tool; and (2) linking existing nodes together with the alppropriate plan instances and relations. The first of these lasks is performed using a controlled natural langlage interface while the second is done with a dialog box mechanism.

Specifying the nodes appearing in the task model involves specifying a full complex of lin guistic entitios and rolo-fillers (o.g., actors, actecs, destinations). Because this structuro may inchude many instances interconnected in potentially un intuitive ways, we have provided a Controlled Natural langrage (CNI) interface for the anthor. 
The interface is shown in Figure 3. This interface allows the author to work in terms of sentences rather than in terms of interconnected graphs. The figure, for example, shows the author in the process of specifying the node Save A Document. The top line of text (reader save [information]) shows the current state of the CNL spccification. Words in brackets must be further specificd. This is done by clicking on the word and selecting the appropriate pattern from a list of possible expansions. In the figure, the author has clicked on [information] and is presented with a list of the types of information from which [document] can be selected. This process is driven by a controlled natural language grammar which specifies possible expansions at each point of the derivation. The bottom line of text presents a fully expanded default at each point in the derivation. In the figure, this CNL text is "reader save current document" which could be expressed in English in a number of ways including "Save the current document" and "To save the document".

Once the action nodes of the graph have been created, or perhaps while they are being created, the author has the ability to link them together using a set of predefined procedural relations: goal, precondition, sub-action, side-effect, warning, and cancellation. This is done with a graphical outlining mechanism. This mechanism allows authors to drag actions from the ACTIONS pane and drop them on the various procedural relation slots in the workspace pane, or, alternatively, to create new actions to fill the slots. The result is a procedural hierarchy such as the one shown in Figure 1.

This interface allows the author to specify the procedure in several ways. They may start from the main goal and work down the structure, or they may start by specifying all the low-levol actions and object and work up the structure.

\subsubsection{The Knowledge Grapher}

The Knowledge Grapher prevents the author from losing orientation by maintaining the current state of the procedural structure in graphical form. This form is like that shown in Figure 1. Because the nodes are mouse-sensitive, it allows the author to initiate construction and maintenance functions by clicking on the appropriate nodes in the graph. Authors can also invoke the drafting tool from the graph.

\subsubsection{The Draft Text Viewer}

The author may draft multilingual instructions on any portion of the procedural structure at any point in the specification process. This task is performed by the Drafting Tool which is briefly described in the next section. This tool produces a draft of the instructions in English and French. These are presented to the author by the Draft Text Vicwer. The presented text is mousesensitive, allowing the author to access the knowledge base entry for selected part of the toxt. In this way, the author can modify the underlying knowledge base while working from the text. In some cases the writer will decide to modify the generated text rather than the underlying knowledge. For this purpose, a text editor is currently provided.

\subsection{The Drafting Tool}

When the author initiates the Drafting Tool (see Figure 2), DRAFI'ER calls the Text Planner with the discourse goal: make the user competent to perform the action specified by the author. The Text Planner selects the content appropriate for the instructions and builds a deep representation of the text to be generated. This portion of the text planning task is done by the text planner developed by Moore and Paris (1993). The Text Planner then specifies the detailed elements of the sentence structure. This portion of the task is done by a descendent of IMAGENE (Vander Linden and Martin, 1995).

Once complete, the text plans are passed to the Tactical Generator which generates the actual text in English and French. This task is performed by the English and French resources of the KometPenman Multi-Lingual development, environment, (KPML) (Bateman, 1995), The drafts generated for the example procedure are shown in Figure 4.

In these toxts, we see that the main user goal, that of saving a document, is given as a title to the series of steps. Then, the steps to be performed to achieve this goal are given. More detail on the drafting process can be found clsewhere.

\section{Summary}

In this paper, we have shown that the knowledge base required to produce user-oriented documentation automatically can be partially obtained from user interface tools and then augmented appropriately by technical authors. We prosented a multilingual drafting tool which exploits output from an interface design tool and provides flexible support to technical authors for augmenting the interface model thus obtained in order to build the task model required to generate documentation. We argued that software documentation is thus an attractive and realistic application for natural language generation. In our current, work, we are extending the percentage of the model that can be 


\begin{tabular}{l|l|} 
[OK] Return] Cancel] \\
\hline $\begin{array}{l}\text { [appointment] } \\
\text { name of [document] } \\
\text { description of [appointment] } \\
\text { date of [appointment] } \\
\text { start time of [appointment] } \\
\text { stop time of [appointment] }\end{array}$ \\
\hline
\end{tabular}

Figure 3: 'The Controlled Natural Language Interface

\section{To Save a Document}

1. Choose Save from the file menu.

\section{-OR-}

Click on the Save icon.

Word displays the Save As dialog box.

2. Type the document name in the Save Current Document As field.

3. Open the folder of the document.

4. Choose the Save button.

You can quit the Save As dialog box by choosing the Cancel button.

\section{Enregistrement d'un document}

1. Choisir Enrcgistrer dans le menu Fichier.

OU BIEN

Cliquer sur l'icone Enregistrer.

Word aflichera la zone de dialogue Enregistrer Sous.

2. Introduire le titre du document dans la zone de texte Enregistrer le Document.

3. Ouvrir le fichier du clocument.

4. Choisir le bouton Enregistrer.

Vous pouve\% quitter la zone de dialogue Enregistrer

Sous en choisissant le bouton Annuler.

Figure 4: Generated English and Trench Drafts

built automatically, so as to increase the usefulness of the system and its potential marketability. Wo are also planning to evaluate the system with technical authors.

\section{References}

John A. Bateman. 1995. KPML: The KOMETPenman (Multilingual) Development Environment. Technical report, Institut für Integrierte Publikations- und Informationssysteme (IPSI), GMD, Darmstadt, July. Release 0.8.

S. K. Card, T. P. Moran, and A. Newell. 1983. The Psychology of Human-Computer Interaction. Lawrence Earlbaum Associates, Hillsdale, N.J.

Robert MacGregor. 1988. A Deductive Pattern Matcher. In Proceedings of the 1988 Conference on Artificial Intelligence, St Paul, MN, August. American Association of Artificial Intelligence.

Johanna D. Moore and Cécile L. Paris. 1993. Planning text for advisory dialogues: Capturing intentional and rhetorical information. Computational Linguistics, 19(4):651-694.

Roberto Moriyon, Pedro Szekely, and Robert Neches. 1994. Automatic generation of help from interface design models. In CII'94 Proceedings, Boston, Mass. Computer Human Interactions.

Cécile Paris, Keith Vander Linden, Markus Fischer, Anthony Hartley, Lyn Pemberton, Richard Power, and Donia Scott. 1995. $\Lambda$ support, tool for writing multilingual instructions. In IJCAI-95, pages 13981404.

Angel R. Puerta and Pedro Szekely. 1994. Modelbased interface development. CHI-94 'Tutorial Notes.

Earl D. Saccrdoti. 1977. A Structure for Plans and Behavior. Elsevior, New York.

Keith Vander Linden and James H. Martin. 1995. Expressing local rhetorical relations in instructional text: $\Lambda$ casc-study of the purpose relation. Computational Linguistics, 21(1):29-57, March.

ParcPlace Systerns, Inc., 999 E. Arques Avenue, Sunnyvale, CA 94086-4593, 1994. The VisualWorks Documentation. 